\title{
Correction to: Peripheral Th17/Treg imbalance in elderly patients with ischemic stroke
}

\author{
Sanam Dolati ${ }^{1,2,3,4} \cdot$ Majid Ahmadi $^{1,2} \cdot$ Mohammad Khalili $^{5} \cdot$ Ali Akabar Taheraghdam $^{5} \cdot$ Homayoon Siahmansouri $^{2,4}$. \\ Zohreh Babaloo $^{4} \cdot$ Leili Aghebati-Maleki $^{6} \cdot$ Farhad Jadidi-Niaragh $^{2,4} \cdot$ Vahid Younesi $^{7} \cdot$ Mehdi Yousefi $^{2,4,8}$
}

Published online: 20 February 2018

(C) Springer-Verlag Italia S.r.l., part of Springer Nature 2018

\section{Correction to: Neurol Sci \\ https://doi.org/10.1007/s10072-018-3250-4}

In the original article the terms ROR $\gamma \mathrm{t}$ and TGF- $\beta$ were misspelled throughout the text. They should read ROR $\gamma \mathrm{t}$ and TGF- $\beta$ instead. We apologize for the inconvenience. The original article has been corrected.

The online version of the original article can be found at https://doi.org/ $10.1007 / \mathrm{s} 10072-018-3250-4$

\section{Mehdi Yousefi}

Mehdi_yusefi@yahoo.com

1 Aging Research Institute, Tabriz University of Medical Sciences, Tabriz, Iran

2 Drug Applied Research Center, Tabriz University of Medical Sciences, Tabriz, Iran

3 Student's Research Committee, Tabriz University of Medical Sciences, Tabriz, Iran

4 Department of Immunology, School of Medicine, Tabriz University of Medical Sciences, Tabriz, Iran

5 Neurosciences Research Center, Tabriz University of Medical Sciences, Tabriz, Iran

6 Immunology Research Center, Tabriz University of Medical Sciences, Tabriz, Iran

7 Pishtaz Teb Zaman Diagnostics, Tehran, Iran

8 Department of Immunology, Faculty of Medicine, Tabriz University of Medical Sciences, Tabriz, Iran 